\title{
Frequency multiplication using coherent radiation of a "snake" beam
}

\author{
G. Stupakov \\ SLAC National Accelerator Laboratory, Menlo Park, California 94025, USA
}

(Received 9 April 2012; published 18 January 2013)

\begin{abstract}
We propose to use a relativistic electron beam which has a shape of a wiggle to generate coherent radiation. We show that such a beam can generate not only radiation with a wavelength equal to the period of the wiggle, but also higher harmonics of that period. Specific calculations are carried out for undulator radiation. Requirements for the transverse size of the beam are obtained.
\end{abstract}

DOI: 10.1103/PhysRevSTAB.16.010702

PACS numbers: 41.60.Cr, 41.75.Fr, 87.56.bd

The remarkable success of the first $\mathrm{x}$-ray free electron lasers (FELs) such as Linac Coherent Light Source at SLAC in the U.S. [1] and SACLA in Japan [2], as well as expected performance of several future such projects, is based on the fundamental physical principle of coherent radiation. A relativistic, high-brightness electron beam in an FEL propagates through a magnetic system with oscillating along the beam path magnetic field (an undulator), and develops an instability that leads to bunching of the particles in the beam on a scale equal to the wavelength of radiation from the undulator. If the undulator is long enough, in its final part, the depth of the modulation can approach $100 \%$ (a so-called saturation regime) and results in coherent radiations of $\mathrm{x}$ rays with the peak power of tens of even a few hundred of gigawatts.

The mechanism that leads to the microbunching of the beam in the undulator is a self-amplified spontaneous emission (SASE) $[3,4]$. It is an instability that starts from an initial random noise in the beam, and results in the FEL radiation which, while having good transverse coherent properties, has a rather poor temporal coherence. There are currently several actively pursued approaches aimed at improving the temporal coherence of FELs. Some of them rely on the application of an initial monochromatic "seed" for the instability, either in the form of an electromagnetic wave generated using the high harmonic generation technique [5] or by modulating the current of the beam through high-gain harmonic generation [6,7] or echo-enabled harmonic generation $[8,9]$. The two last techniques rely on the frequency multiplication of the current modulation in the beam generated with the help of a conventional laser pulse.

In this paper we propose a novel mechanism of generating the high harmonics of coherent radiation which does not involve microbunching (that is modulation of the current) of the beam. In contrast to the traditional approach, it uses a bunch which has a special geometrical shape of a

Published by the American Physical Society under the terms of the Creative Commons Attribution 3.0 License. Further distribution of this work must maintain attribution to the author(s) and the published article's title, journal citation, and DOI. wiggle, as shown in Fig. 1. For brevity, we will call such a beam a "snake" one. As we show in this paper, a remarkable property of such a beam is that it can coherently radiate not only at wavelength of the wiggle $\lambda_{0}$, but also at its higher harmonics $\lambda_{0} / h$, where $h$ is an integer number.

To demonstrate the basic mechanism of the radiation, we first consider a line-charge beam schematically shown in Fig. 1 with the charge distributed along the line $x=$ $a \sin (x z)$, where $x=2 \pi / \lambda_{0}$. Denote the one dimensional particle density (that is the number of particles per unit length along $z$ ) by $\rho(z)$. Our main interest will be in the case $\rho=$ const (no microbunching); however, for comparison with the radiation of a microbunched beam we start with a general case of arbitrary $\rho(z)$. For now we assume that the "thickness" of the line beam is small and neglect it. The beam radiation is detected at a location far from the undulator.

To calculate the beam radiation, we first consider radiation of a single electron in the beam. The vector potential $\boldsymbol{A}$ of radiation at frequency $\boldsymbol{\omega}$ in the far zone can be written as [10]

$$
\boldsymbol{A}=\frac{e}{c R_{0}} e^{i \omega R_{0} / c} \int_{-\infty}^{\infty} \boldsymbol{v}(t) e^{i k\left[c t-n \cdot r_{0}(t)\right]} d t,
$$

where $R_{0}$ is the distance from the undulator to the observer, $k=\omega / c, e$ is the electron charge, $c$ is the speed of light, $\boldsymbol{v}(t)$ and $\boldsymbol{r}_{0}(t)$ are the velocity and the radius vector of the moving electron as functions of time, and $\boldsymbol{n}$ is the unit vector in the direction of radiation. The spectral intensity of radiation (energy radiated per unit $\omega$ per unit solid angle) is

$$
P(\omega, \boldsymbol{n})=\frac{\omega^{2} R_{0}^{2}}{4 \pi^{2} c}|\boldsymbol{n} \times \boldsymbol{A}|^{2} .
$$

To find radiation of the beam we need to sum (1) over all electrons in the bunch. For a line-charge distribution of particles, we denote by $\boldsymbol{r}(t, \zeta)$ the radius vector of the electron located initially (at $t=0$, before the entrance to the undulator) at the longitudinal coordinate $z=\zeta$; the coherent radiation is then given by an integral of $\boldsymbol{A}$ over $\zeta$. We assume that electrons are moving with constant velocity $v_{z}$ through the undulator. Neglecting a weak focusing effect of the undulator magnetic field, we have 


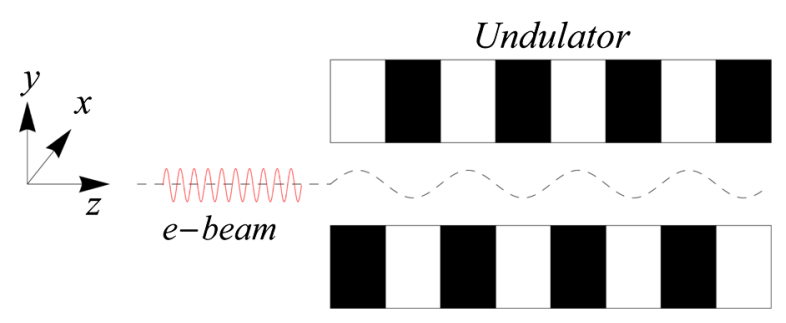

FIG. 1. Cartoon of a thin snake beam at the entrance to an undulator. The beam charge is localized in the horizontal plane on the line $x=a \sin \left(2 \pi z / \lambda_{0}\right)$. The broken line shows the trajectory of the beam. The relative length of the beam and the period of the undulator are not to scale.

$$
\boldsymbol{r}(t, \zeta)=\boldsymbol{r}_{0}\left(t-\zeta / \boldsymbol{v}_{z}\right)+\hat{\boldsymbol{x}} a \sin (x \zeta),
$$

which means that every electron is moving along the same trajectory, shifted appropriately in the $x$ direction and delayed by the time interval $\zeta / v_{z}$. In what follows we assume a relativistic beam and replace $v_{z}$ by $c$ in (3). Substituting (3) to (1), it is easy to see that the vector potential for the whole beam, $\boldsymbol{A}_{b}$, is

$$
\boldsymbol{A}_{b}=F(\omega, \boldsymbol{n}) \boldsymbol{A},
$$

where $\boldsymbol{A}$ is given by (1) and the form factor $F$ is

$$
F(\omega, \boldsymbol{n})=\int \rho(\zeta) e^{i k\left[\zeta-n_{x} a \sin (x \zeta)\right]} d \zeta
$$

The intensity of the coherent beam radiation $P_{b}$ is

$$
P_{b}=|F(\omega, \boldsymbol{n})|^{2} P(\omega, \boldsymbol{n}),
$$

with $P$ given by (2). In addition to (6) the beam also radiates incoherently with the incoherent radiation power equal to $N P(\omega, \boldsymbol{n})$ where $N$ is the number of particles in the beam.

To contrast our proposal with the traditional mechanism of coherent radiation based on a density modulated beam, we first consider the case of a straight bunch $(a=0)$ of length $l$ having a sinusoidal density modulation with the period $\lambda_{1} \ll l$,

$$
\rho(\zeta)=\frac{Q}{e l}[1+2 b \sin (\tilde{x} \zeta)], \quad \text { for }-\frac{l}{2}<z<\frac{l}{2},
$$

where $Q$ is the bunch charge, $b$ is a so-called bunching factor, and $\tilde{x}=2 \pi / \lambda_{1}$. Apart from the frequency range $\omega \sim c / l$ associated with the finite bunch length, the form factor $F$ is localized in the vicinity of the frequency $\omega_{*}=$ $c \tilde{x}$, and the peak has a relative width $\Delta \omega / \omega \sim \lambda_{1} / l$. Replacing the narrow peak profile around $\omega_{*}$ by a delta function, one finds from (5) $|F(\omega)|^{2}=b^{2} \mathcal{F}^{2}(\omega)$ with

$$
\mathcal{F}^{2}(\omega)=2 \pi l \frac{I_{b}^{2}}{c e^{2}} \delta(\omega-c \tilde{x}),
$$

where we introduced the beam current $I_{b}=Q c / l$. We see that the radiation wavelength is equal to the modulation period of the beam and the intensity is proportional to the square of the beam current indicating the coherent nature of the radiation.

Let us now turn our attention to the main subject of this paper - the case without density modulation, $\rho=$ const in (5), but a sinusoidally modulated shape instead, $a>0$. Using the expansion $e^{i a \sin x}=\sum_{h} J_{h}(a) e^{i h x}$, one finds from (5)

$$
F=\frac{Q}{e l} \int_{-l / 2}^{l / 2} d \zeta e^{i k \zeta} \sum_{h=-\infty}^{\infty} e^{i h x \zeta} J_{h}\left(-k a n_{x}\right)
$$

Analogously to the derivation of (8), one now finds

$$
|F|^{2}=\sum_{h=0}^{\infty}\left|F_{h}\right|^{2}
$$

with

$$
\left|F_{h}\right|^{2}=2 \pi l \frac{I_{b}^{2}}{c e^{2}} J_{h}^{2}\left(\operatorname{kan}_{x}\right) \delta(\omega-\operatorname{ch} x) .
$$

In contrast to (8), we see that coherent radiation now occurs not only at the fundamental harmonic, $h=1$, but also at all higher harmonics with the radiation wavelength $\lambda=\lambda_{0} / h$.

While low harmonics $h \sim 1$ of coherent radiation are of certain interest for some applications, the most attractive property of radiation of a snake beam lies in the presence of high harmonics, $h \gg 1$. To simplify the subsequent analysis we assume a plane undulator [11] with a small undulator parameter, $K \ll 1$, where $K=\lambda_{u} e B_{0} / m c, \lambda_{u}$ is the undulator period, and $B_{0}$ is the amplitude value of the magnetic field. To characterize the angular properties of the radiation, we will use the polar angle $\theta$ relative to the $z$ axis, and the azimuthal angle $\phi$ of vector $n$ with respect to the horizontal plane $x z$; then $\boldsymbol{n}=$ $(\sin \theta \cos \phi, \sin \theta \sin \phi, \cos \theta)$. For an undulator with a large number of periods $N_{u}$, for a given $\theta$, the function $P$ is a narrow function of frequency $\omega$ of width $\Delta \omega / \omega \sim$ $1 / N_{u}$. In what follows we replace the narrow peak in $P$ by the delta function, then $P$ is given by the following expression [11]:

$P(\omega, \theta, \phi)=P_{0} \frac{\left(1+\gamma^{2} \theta^{2}\right)^{2}-4 \theta^{2} \gamma^{2} \cos ^{2} \phi}{\left(1+\gamma^{2} \theta^{2}\right)^{5}} \delta\left(\omega-\frac{\omega_{0}}{1+\gamma^{2} \theta^{2}}\right)$,

where $\omega_{0}=2 \gamma^{2} k_{u} c$ and $P_{0}=e^{4} \gamma^{2} B_{0}^{2} L_{u} / \pi m^{2} c^{4}$ with $L_{u}$ the undulator length. Given that the intensity of the radiation at harmonic $h$ is the product of (11) and (12), and they both involve delta functions of frequency, we find that the coherent radiation propagates at an angle $\theta$,

$$
\theta=\frac{1}{\gamma} \sqrt{\frac{\omega_{0}}{c x h}-1}
$$

It follows from (13) that, for a given $x$ and $\omega_{0}$, the allowable harmonics $h$ are in the range $1 \leq h<\omega_{0} / c x$. 


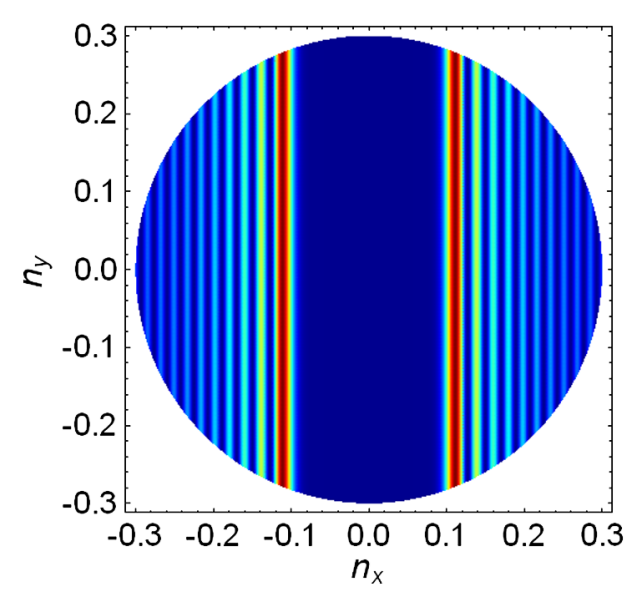

FIG. 2. Density plot of the function $J_{h}^{2}\left(h x a n_{x}\right)$ in the $n_{x}, n_{y}$ plane.

For large values of $h$ the Bessel function $J_{h}(x)$ has a maximum equal to $0.67 / h^{1 / 3}$ at $x \approx h$. To maximize $P_{b}$ the directionality of the form factor $\left|F_{h}\right|^{2}$ should be matched with that of the angular distribution of radiation of a single particle. For illustration, Fig. 2 shows the distribution of the angular factor $J_{h}^{2}\left(h x a n_{x}\right)$ in (11) in the transverse plane perpendicular to the $z$ axes for $h=20$ and $x a=10$. The maximal values of this function are localized along the vertical stripes $n_{x} \approx 1 / x a$. The closest approach to the $z$ axes of the brightest line occurs for $\phi=0$, and corresponds to $\theta \approx 1 / x a$. Equating this value of $\theta$ to that given by (13) and recalling that $c x h=\omega$, we find the relation between the required amplitude of wiggles $a$, the radiation frequency $\omega$, and the harmonic number $h$ :

$$
a \approx \frac{\operatorname{ch} \gamma}{\omega^{1 / 2} \sqrt{\omega_{0}-\omega}} .
$$

For relativistic beams, $\gamma \gg 1$, this amplitude is large in comparison with the period of modulation $2 \pi / x$.

Let us now calculate the integrated over frequency and angle total energy of radiation at harmonic $h$,

$$
\mathcal{P}_{h}=\int d \omega \theta d \theta d \phi\left|F_{h}\right|^{2} P
$$

where we used the smallness of $\theta$ to replace $\sin \theta \rightarrow \theta$. To compare the power of the snake beam radiation with that of sinusoidally bunched beam, we will introduce the effective bunching ratio for the snake beam. It is defined in such a way that its square $b_{\text {eff }}^{2}$ is equal to the ratio of $\mathcal{P}_{h}$ to an integrated power of the microbunched beam (7) with a unit value of $b$ (assuming $\tilde{x}=h x$, so that both beams radiate at the same frequency),

$$
b_{\text {eff }}^{2}=\mathcal{P}_{h}\left(\int d \omega \theta d \theta d \phi \mathcal{F}^{2}(\omega) P\right)^{-1},
$$

where $\mathcal{F}$ is defined in (8). A straightforward calculation yields

$$
\begin{aligned}
b_{\mathrm{eff}}^{2}(h, A, X)= & \frac{1}{2 \pi} \int_{0}^{2 \pi} d \phi \frac{1-4 X(1-X) \cos ^{2} \phi}{2 X^{2}-2 X+1} \\
& \times J_{h}^{2}[A \cos \phi \sqrt{X(1-X)}]
\end{aligned}
$$

where we now use dimensionless variables $X=\omega / \omega_{0}$ and $A=\omega a / c \gamma$. This equation can be simplified in the limit $h \gg 1$ and $X$ close to 1 . Analysis shows that in this limit $b_{\text {eff }}$ is maximized if the amplitude of the wiggle is chosen such that

$$
A \approx \frac{h+1.2 h^{1 / 3}}{\sqrt{1-X}},
$$

and the maximized value of $b_{\text {eff }}$ is

$$
b_{\text {eff }} \approx \frac{0.64}{\sqrt{h}} \sqrt{3 X-2} .
$$

The last equation gives the scaling $b_{\text {eff }} \propto 1 / \sqrt{h}$.

For illustration of typical radiation power that can be achieved in practice with the proposed system, let us consider a relativistic snake beam with $\gamma=600(300 \mathrm{MeV}$ electron energy) that is created with the wiggle period $\lambda_{0}=0.2 \mu \mathrm{m}$ with the aim to generate the harmonic $h=$ 20. We choose $X=0.9$ for which Eq. (18) gives $A=0.75$, and hence the amplitude of the wiggles $a=c \gamma A / \omega \approx$ $72 \mu \mathrm{m}$. The effective bunching factor (19) is $b_{20} \approx 0.12$. The value $X=0.9$ means that the undulator should be tuned to the fundamental wavelength (of radiation propagating on axis) equal to $9 \mathrm{~nm}$ (for $K=1$ and the beam energy specified above this would require the undulator period of $\lambda_{u}=4 \mathrm{~mm}$ ). Assuming a $2 \mathrm{~m}$ long undulator and beam current of $I_{b}=1 \mathrm{kA}$ (typical for modern x-ray FELs) this beam will radiate $100 \mathrm{MW}$ at $10 \mathrm{~nm}$ wavelength. Note that in the above calculation we took $K=1$, while our derivation actually assumed $K \ll 1$-hence, our numerical example should be considered as a rough estimate only.

We now consider the effect of the finite thickness of the beam that was neglected above. In addition to the term $\hat{\boldsymbol{x}} a \sin (x \zeta)$ in (3) which determines the wiggle shape of the beam, we now need to take into account additional offsets $X$ and $Y$ for each particle due to the finite transverse size of the beam,

$\boldsymbol{r}(t, \zeta, X, Y)=\boldsymbol{r}_{0}\left(t-\zeta / v_{z}\right)+\hat{\boldsymbol{x}} a \sin (x \zeta)+\hat{\boldsymbol{x}} X+\hat{\boldsymbol{y}} Y$,

where we will assume that the coordinates $X$ and $Y$ satisfy a Gaussian distribution function $\left(2 \pi \sigma_{x} \sigma_{y}\right)^{-1} \times$ $e^{-Y^{2} / 2 \sigma_{y}^{2}-X^{2} / 2 \sigma_{x}^{2}}$ with the rms beam sizes $\sigma_{x}$ and $\sigma_{y}$ in the $x$ and the $y$ direction, respectively. Repeating the derivation of (5), we now arrive at the following expression for the form factor $F$ :

$$
\begin{aligned}
F(\boldsymbol{\omega}, \boldsymbol{n})= & \frac{1}{2 \pi \sigma_{x} \sigma_{y}} \int_{-l / 2}^{l / 2} d \zeta \int_{-\infty}^{\infty} d Y \int_{-\infty}^{\infty} d X e^{-Y^{2} / 2 \sigma_{y}^{2}-X^{2} / 2 \sigma_{y}^{2}} \\
& \times e^{i k\left[\zeta-n_{x} a \sin (x \zeta)-n_{x} X-n_{y} Y\right]},
\end{aligned}
$$


which after all integrations gives the following expression for $\left|F_{h}\right|^{2}$ :

$$
\left|F_{h}\right|^{2}=2 \pi l \frac{I_{b}^{2}}{c e^{2}} J_{h}^{2}\left(k a n_{x}\right) \delta(\omega-\operatorname{ch} x) e^{-k^{2}\left(\sigma_{x}^{2} n_{x}^{2}+\sigma_{y}^{2} n_{y}^{2}\right) / 2}
$$

We see that the finite transverse beam size leads to an exponential suppression of $\left|F_{h}\right|^{2}$ and as such imposes an important constraint on the allowable transverse size of the beam. For a rough estimate we can take $\theta \sim 1 / \gamma$ [see Eq. (13)] and $n_{x} \sim \theta$, which gives the following constrain on the transverse size in the $x$ direction: $\sigma_{x} \lesssim$ $\gamma \lambda_{0} / 2 \pi h$. The requirement for the transverse size in $y$ is milder because $n_{y} \sim \theta \phi$, and the angle $\phi$ is small.

While we considered a particular case of a weak undulator as a radiator, as the derivation shows, the form factor (22) is actually valid for other types of radiation-e.g., synchrotron radiation or transition radiation from a metallic foil-if in the process of radiation particles move on a parallel trajectories shifted in the transverse direction, as described by (3). For each case, the function $P$ in (6) will be different corresponding to the specific type of radiation. Note that our choice that the beam has a shape of a wiggle in the $x-z$ plane is not crucial: the wiggle can also lie in the $y-z$ plane (with minor changes in the final result), or any other plane passing through the $z$ axis.

Note also that a beam of helical shape will be even more efficient because its form factor being axisymmetric will have a better overlap with the angular distribution of the undulator radiation.

There is an important issue of how a snake beam with a large $a / \lambda_{0}$ ratio can be generated in practice. A detailed design of a system for generation of such a beam is beyond the scope of this paper. A possible approach might be to modulate the beam energy using an external laser by sending it through a modulator-undulator tuned to the laser frequency (see, e.g., [7]). If such a beam is then sent through a dispersive section located after the modulator particles will shift in the horizontal plane in proportion to their energy, and the beam shape will become a wiggle. It is important in the design of such a system to minimize the longitudinal displacements of particles which will tend to smear out the profile of the beam. Unfortunately, simple estimates show that the typical emittance of currently available electron beams is not small enough to generate the snake beam with required properties. Generation of such beams remains an open challenge, but we hope that the fast development of beam generation techniques (e.g., such as the nanocathode field-emission array [12]) can make it realistic in the future.

\section{ACKNOWLEDGMENTS}

The author would like to thank M. Zolotorev for useful discussions and encouragement.

[1] P. Emma et al., Nat. Photonics 4, 641 (2010).

[2] H. Tanaka, in Proceedings of the 2011 FEL Conference (Shanghai, China, 2011) [http://accelconf.web.cern.ch/ accelconf/FEL2011/index.htm].

[3] A. Kondratenko and E. Saldin, Part. Accel. 10, 207 (1980).

[4] R. Bonifacio, C. Pellegrini, and L. M. Narducci, Opt. Commun. 50, 373 (1984).

[5] T. Tanikawa, G. Lambert, T. Hara, M. Labat, Y. Tanaka, M. Yabashi, O. Chubar, and M. E. Couprie, Europhys. Lett. 94, 34001 (2011).

[6] R. Bonifacio, L.D.S. Souza, P. Pierini, and E.T. Scharlemann, Nucl. Instrum. Methods Phys. Res., Sect. A 296, 787 (1990).

[7] L. Yu, Phys. Rev. A 44, 5178 (1991).

[8] G. Stupakov, Phys. Rev. Lett. 102, 074801 (2009).

[9] D. Xiang and G. Stupakov, Phys. Rev. ST Accel. Beams 12, 030702 (2009).

[10] L. D. Landau and E. M. Lifshitz, The Classical Theory of Fields, Course of Theoretical Physics (Pergamon, London, 1979), Vol. 2, 4th ed.

[11] A. Hofmann, The Physics of Synchrotron Radiation (Cambridge University Press, Cambridge, England, 2004).

[12] W. S. Graves, F. X. Kärtner, D. E. Moncton, and P. Piot, Phys. Rev. Lett. 108, 263904 (2012). 